\title{
No-boundary Codimension-two Braneworld
}

\author{
Zhong Chao Wu \\ Dept. of Physics \\ Zhejiang University of Technology \\ Hangzhou 310032, China
}

\begin{abstract}
The quantum creation probability and entropy of a 2-codimensional braneworld are calculated in the framework of no-boundary universe. The entropy can take an arbitrarily large value as the brane tensions increase, in violation of the conjectured " $N$-bound" in quantum gravity, even for a 4-dimensional ordinary universe.

PACS number(s): 04.50.+h; 98.80.Cq; 11.10.Kk; 04.65.+c

Keywords: braneworld, quantum cosmology, constrained gravitational instanton, "N-bound" conjecture

e-mail: zcwu@zjut.edu.cn,
\end{abstract}


In recent years, the so-called braneworld has attracted a lot of attention. Here the Standard Model fields are confined in the 4-dimensional brane, which is embedded in a higher dimensional bulk space. The extra dimensions can only be perceived through gravitational interactions. They need not necessarily be small and may even be on the scale of millimeters. This concept provides a possible solution to the hierarchy problem; that is the large difference between the Planck and electroweak scales [1]. Randall and Sundrum [2] showed that the 4-dimensional Newton gravity can be reproduced in the brane even in the presence of an infinitely large extra dimension. In the $R S$ model the bulk spacetime is described by a 5-dimensional Anti-de Sitter spacetime.

The interest of brane cosmology was inspired by string theory. Horava and Witten [3] suggested an 11-dimensional spacetime model. The eleventh dimension is compactified on an orbifold with $Z_{2}$ symmetry, while 6 dimensions can be compactified on a Calabi-Yau manifold in the usual way.

On the other hand, it is believed that the quantum state of the universe is defined by the ground state in no-boundary universe [4]. The wave function of the universe is expressed as the path integral over all Euclidean metrics and matter fields on them. The contribution of an instanton solution dominates the path integral. Therefore, at the $W K B$ level, the Lorentzian universe can be obtained via analytic continuation from the instanton. Even though the no-boundary proposal works only for a closed universe, one can still analytically continue a complex instanton to obtain an open universe, at the same $W K B$ level of approximation [5].

Some work have been done on the quantum creation of braneworlds [6]. Some of this work has been based on the $R S$ model, unfortunately, there does not exist a compact seed instanton for it. Although the most effort has been paid to the 1-codimensional braneworld, higher codimensional models are attracting interest recently [7]. It is hoped that some of 2-codimensional models provide a distinctive step toward understanding the smallness of the cosmological constant. In this paper we shall discuss a creation scenario of 2-codimensional braneworld.

For generality and simplicity, the bulk dimension is not specified here. The Euclidean action for a $n$-dimensional bulk spacetime $M$ with a brane $B$ is

$$
I=-\frac{1}{16 \pi} \int_{M}(R-2 \Lambda)-\frac{1}{8 \pi} \int_{\partial M} K+\frac{1}{8 \pi} \int_{B} \sigma,
$$

where the $n$-dimensional Newton constant is set to be $1, \Lambda$ is the bulk cosmological constant, the scalar curvature $R$ and the trace of the second fundamental form $K$ are defined with respect to the bulk. The boundary $\partial M$ may be degenerate and then $K$ can take distribution form. This is the case 
for the boundary associated with the 2-codimensional branes. We have assumed that there is no matter content in the bulk except for a cosmological constant $\Lambda$, the only energy-momentum tensor of the brane is represented by the tension $\sigma$.

We first consider the Schwarzschild-like instanton

$$
d s^{2}=f(r) d \psi^{2}+f(r)^{-1} d r^{2}+r^{2} d \Omega_{n-2}^{2},
$$

where

$$
f(r)=k-\frac{2 m}{r^{n-3}}-\frac{2 \Lambda r^{2}}{(n-1)(n-2)},
$$

where $m$ is a parameter. $d \Omega_{n-2}^{2}$ is the metric of an unit $n-2-$ dimensional Einstein manifold, called the base manifold. If it is restricted to be maximally symmetric, then it must be either a $n-2$-dimensional sphere, a plane or a hyperboloid labelled by $k=1,0,-1$, respectively. The hyperboloid and plane can be compactified using their discrete isometry subgroup. The sphere can also be replaced by its lens space.

In general, there are $n-1$ horizons $r_{i}$ associated with the zeros of $f(r)$. The surface gravity at the horizon $r_{i}$ is

$$
\kappa_{i}=\frac{1}{2}\left|\frac{d f(r)}{d r}\right|_{r=r_{i}} .
$$

One can construct a compact manifold by periodically identifying $\psi$ coordinate between two neighboring horizons $r_{l} \leq r \leq r_{k}$, with $f(r) \geq 0$. Since the motivation of this article is to discuss the braneworld, there is no restriction for the signature of the parameter $m$. Therefore, for $k=0,-1$, in order for the two horizons to exist, there is no restriction for the signature of the cosmological constant either.

If one chooses the period $\beta_{l}=2 \pi \kappa_{l}^{-1}$, then the conical singularity at the horizon $r_{l}$ can be regularized. However, in general the two surface gravities are distinct, therefore one cannot construct a regular instanton.

One can keep the identified coordinate $\psi$ of metric (2) intact and analytically continue the base space $d \Omega_{n-2}^{2}$. In this case, the base space becomes a $n-2$-dimensional de Sitter, Minkowski, Anti-de Sitter or their respective lens spacetime. The analytic continuation from the sphere $S_{n-2}$ of the base space to the $n-2$-dimensional de Sitter space is well known [4]. From the hyperboloid $H_{n-2}$ of the base space

$$
d s^{2}=d \theta^{2}+\sinh ^{2} \theta d S_{n-3}^{2}=d \theta^{2}+\sinh ^{2} \theta\left(d \kappa^{2}+\sin ^{2} \kappa d S_{n-4}^{2}\right)
$$


one can obtain the $n-2$-dimensional Anti-de Sitter space

$$
d s^{2}=-d t^{2}+\cos ^{2} t\left(d \phi^{2}+\sinh ^{2} \phi d S_{n-4}^{2}\right)=-d t^{2}+\cos ^{2} t d H_{n-3}^{2},
$$

by the analytic continuation $t=i \theta+\pi / 2$ and $\phi=i \kappa$, where $d S_{d}^{2}$ and $d H_{d}^{2}$ represent the metrics of unit $S_{d}$ and $H_{d}$, respectively.

This technique is called double analytic continuation, which was first introduced by Witten to study the instability of the Kaluza-Klein vacuum [8]. The obtained Lorentzian manifolds, dubbed "bubbles of nothing", can be considered as time dependent backgrounds for string theory [9][10]. It is noted that the backgrounds must be regular and no singularity is allowed. Therefore, in the literature, the technique of double continuation is applied only to a black hole with asymptotically flat or Anti-de Sitter background.

The motivation of this paper is different. We are going to use the same seed instanton (2) for braneworld creation. At the $W K B$ level, the braneworld can be obtained by the double continuation. We identify the two conical singularities at the horizons as the 2-codimensional branes embedded in the bulk. The brane in which we are living is a $n-2$-dimensional de Sitter, Minkowski or Anti-de Sitter spacetime.

The Einstein equation should be satisfied everywhere, the brane tension $\sigma$ is the source of the conical singularity. Since the field configuration is invariant with respect to the Lorentz boosts or rotation in the local inertial frame of the base manifold, the tension should behave as a cosmological constant in the base manifold. That is, the pressure $p$ is the negative of the energy density which is $\sigma$. This fact has been accounted in the brane part of the action (1).

Since the conical singularities are allowed for the constructed Euclidean manifold, the identification period $\beta$ can be relaxed from its relation with the surface gravity. The bulk equator of the quantum transition topologically is the product of the space $M_{2}$ associated with coordinates $(\psi, r)$ and an equator of the $n$-2-dimensional base manifold.

We use $M_{l}$ and $M_{k}$ to denote the infinitesimal neighborhoods of the two horizons with the boundary of a constant coordinate $r$, and we use $M^{\prime}$ to represent $M$ minus $M_{l}$ and $M_{k}$. The Euclidean action can be rewritten as

$$
I=I_{l}+I_{k}+\int_{M^{\prime}}\left(\pi^{i j} \dot{h}_{i j}-N H_{0}-N_{i} H^{i}\right) d^{n} x+\frac{1}{8 \pi} \int_{B} \sigma,
$$

where the actions $I_{l}$ and $I_{k}$ are the gravitational actions for $M_{l}$ and $M_{k}$, respectively. The action of 
$M^{\prime}$ has been recast into the canonical form. $N$ and $N_{i}$ are the lapse function and shift vector, $h_{i j}$ and $\pi^{i j}$ are the $n$-1-metric and the conjugate momenta respectively, $H_{0}$ and $H^{i}$ are the Einstein and momentum constraints, and the dot denotes the derivative with respect to the Killing coordinate $\psi$. The manifold satisfies the Einstein equation, and all time derivatives vanish due to the $U(1)$ isometry. Therefore, the integral over $M^{\prime}$ is zero.

Now the action $I_{l}$ or $I_{k}$ can be written

$$
I_{i}=-\frac{1}{16 \pi} \int_{M_{i}}\left({ }^{n} R-2 \Lambda\right)-\frac{1}{8 \pi} \int_{\partial M_{i}}{ }^{n-1} K(i=l, k),
$$

where ${ }^{n} R$ denotes the $n$-dimensional scalar curvature and ${ }^{n-1} K$ is the expansion rate of the boundary. In addition to the action from the boundary of $M_{i}$, the action contribution of the conical singularity can be considered as the degenerate version of the second term. The conical singularity contribution is termed as a deficit "angle". We assume $r_{i}$ to be a single zero of $f(r)$, therefore the horizon does not recede into an internal infinity.

One can apply the Gauss-Bonnet theorem to the 2-dimensional $(\psi, r)$ section of $M_{i}$,

$$
\frac{1}{4 \pi} \int_{\hat{M}_{i}}{ }^{2} R+\frac{1}{2 \pi} \int_{\partial \hat{M}_{i}}{ }^{1} K+\frac{\delta_{i}}{2 \pi}=\chi(i),
$$

where $\hat{M}_{i}$ is the projection of $M_{i}$ onto the 2 -dimensional $(\psi, r)$ section, ${ }^{2} R$ is the scalar curvature on it, ${ }^{1} K$ is the corresponding expansion rate, $\delta_{i}$ is the deficit angle at the apex, and $\chi(i)$ is the Euler characteristic of $\hat{M}_{i}$, which is 1 here. Since the expansion rate of the subspace $r^{2} d \Omega_{n-2}^{2}$ goes to zero at the horizon, ${ }^{n-1} K$ and ${ }^{1} K$ are equal. Comparing Eqs. (8) and (9), one can see that as the circumference of the boundary tends to zero, the action (8) becomes $-\chi(i) A_{i} / 4$, where $A_{i}=r_{i}^{n-2} \Omega_{n-2}$ is the $n-2$-dimensional surface area of the horizon. It is noted that both the volume integral of (8) and the first term of the left hand side of (9) vanish as the boundary approaches the horizon.

Therefore, the total gravitational action is

$$
I_{\text {gravity }}=-\frac{1}{4}\left(A_{l}+A_{k}\right)
$$

That is the negative of one quarter of the sum of the two horizon areas. It is worth emphasizing that the horizon areas are the volumes of the $n-2$-dimensional Euclidean brane manifolds.

In order to find the tension of the brane for the given $\beta$, we first assume that the brane has 2-dimensional finite "thickness", and then let the thickness approach zero later. One can use the 
Gauss-Bonnet theorem again to the 2-dimensional cross section of $\hat{M}_{i}$,

$$
\frac{\alpha_{i}}{2 \pi} \equiv \chi(i)-\frac{1}{2 \pi} \int_{\partial \hat{M}_{i}}{ }^{1} K=\frac{1}{4 \pi} \int_{\hat{M}_{i}}{ }^{2} R
$$

where the deficit angle $\alpha_{i}$ is redefined as the orientation change of a parallel transport of a vector around $\partial \hat{M}_{i}$ instead of that defined at the apex in equation (9). As the thickness is reduced to zero, the limit of $\alpha_{i}$ becomes $\delta_{i}$.

We assume the tension $\sigma_{i}$ is diluted to a tension density $\epsilon_{i}(\psi, r)$ with a finite support ( or the thickness) such that

$$
\sigma_{i}=\int_{\hat{M}_{2}} \epsilon_{i}(\psi, r) .
$$

Since the thickness will be infinitesimal, as long as the relation of the deficit angle and the tension is concerned, the bulk cosmological constant can be ignored. For the same consideration, it is also assumed that the spacetime $\bar{M}_{n}$ near the brane $r_{i}$ can be thought as a direct product of the base manifold $M_{n-2}$ and the cross section $\bar{M}_{2}$

$$
d s^{2}=\gamma_{\alpha \beta} d x^{\alpha} d x^{\beta}+h_{\mu \nu} d x^{\mu} d x^{\nu} \quad(1 \leq \alpha, \beta \leq 2 ; 3 \leq \mu, \nu \leq n),
$$

where $\gamma_{\alpha \beta}$ depends on $\psi, r$ only, and $h_{\mu \nu} r_{i}^{-2}$ is the metric tensor of the base manifold. Inside the brane the energy-momentum tensor is

$$
T_{m}^{q}=\epsilon_{i}(\psi, r) \operatorname{diag}(0,0,1,1, \cdots, 1) \quad(1 \leq q, m \leq n)
$$

The Einstein equation reads

$$
\begin{gathered}
R_{\alpha \beta}-\frac{1}{2} R \gamma_{\alpha \beta}=0, \\
R_{\mu \nu}-\frac{1}{2} R h_{\mu \nu}=-\epsilon_{i}(\psi, r) h_{\mu \nu} .
\end{gathered}
$$

From above one can derive

$$
R=2 \epsilon_{i}(\psi, r)
$$

and

$$
R_{\mu \nu}=0, \quad R_{\alpha \beta}=\epsilon_{i}(\psi, r) \gamma_{\alpha \beta} .
$$

That is in the scale of the brane thickness, the base manifold is nearly Ricci flat, in the sense that the bulk cosmological constant $\Lambda$ is much smaller than $\alpha_{i}$ and so omitted here. It is noted that for the product spacetime, $R_{\alpha \beta}$ remains intact for the reduction $\bar{M}_{n} \longrightarrow \bar{M}_{2}$. 
Substituting (18) and (12) into (11), and letting the thickness approach zero, we see $\epsilon_{i}(\psi, r) \longrightarrow$ $\sigma_{i} \delta\left(r-r_{i}\right) /\left(\pi\left|r-r_{i}\right|\right)$, and

$$
\alpha_{i}=\sigma_{i}
$$

This relation is a generalization of the result of string in 4-dimensional spacetime [11]. Apparently, the codimension of 2 is critical for this argument. The higher co-dimension "thick" brane has been discussed in [12], which are based on an axis symmetry. Our argument is more general.

Now, for our instanton, the deficit angles or the tensions of the two branes are determined by $\beta$

$$
\sigma_{i}=2 \pi-\kappa_{i}|\beta|
$$

Once the tension of one brane is given, both $\beta$ and the tension of the other brane is fixed by eq. (20).

The action of the brane can be written as

$$
I_{\text {brane }}=\frac{1}{8 \pi}\left(\sigma_{l} A_{l}+\sigma_{k} A_{k}\right) .
$$

The total action is the sum

$$
I=I_{\text {gravity }}+I_{\text {brane }}=-\frac{|\beta|}{8 \pi}\left(\kappa_{l} A_{l}+\kappa_{k} A_{k}\right)
$$

Now we consider the Kerr-like instanton [10][13]

$$
\begin{aligned}
d s^{2}=\frac{\Delta_{r}}{\rho^{2}}\left[d \chi+\frac{\alpha}{\Xi} \sin ^{2} \theta d \phi\right]^{2}+ & \frac{\rho^{2}}{\Delta_{r}} d r^{2}+\frac{\rho^{2}}{\Delta_{\theta}} d \theta^{2}+\frac{\Delta_{\theta}}{\rho^{2}} \sin ^{2} \theta\left[\alpha d \chi-\frac{r^{2}-\alpha^{2}}{\Xi} d \phi\right]^{2} \\
& +r^{2} \cos ^{2} \theta d \Omega_{n-4}^{2},
\end{aligned}
$$

where $\alpha$ is the angular momentum parameter for the imaginary time $\chi, d \Omega_{n-4}^{2}$ is a general unit elliptic space, and

$$
\begin{gathered}
\Delta_{r}=\left(r^{2}-\alpha^{2}\right)\left(1-\frac{2 \Lambda r^{2}}{(n-1)(n-2)}\right)-\frac{2 m}{r^{n-5}}, \\
\Delta_{\theta}=1-\frac{2 \Lambda \alpha^{2}}{(n-1)(n-2)} \cos ^{2} \theta, \\
\Xi=1-\frac{2 \Lambda \alpha^{2}}{(n-1)(n-2)}, \\
\rho^{2}=r^{2}-\alpha^{2} \cos ^{2} \theta
\end{gathered}
$$

The Killing coordinate $\chi$ is identified by a period $\beta$ as in the black hole creation scenario [14], i.e one can set $0 \leq \chi \leq \beta|\Xi|^{-1}$. The instanton is constructed from a sector between two neighboring 
horizons $r_{l} \leq r \leq r_{k}$, with $\Delta_{r} \geq 0$. In general, at least one conical singularity arises. Again, these conical singularities are identified as the branes. The Einstein equation should apply to these singularities (branes) as well. By the same argument as for the nonrotating case, the brane tension should equal the deficit angle as in (19)(20).

The double continuation can be carried out through an analytic continuation from $d \Omega_{n-4}^{2}$ into a de Sitter-like spacetime.

Using the same method, the gravitational action in (1) can be derived

$$
I_{\text {gravity }}=-\frac{1}{4}\left(A_{k}+A_{l}\right)
$$

where the horizon area is

$$
A_{i}=\left|\frac{4 \pi\left(r_{i}^{n-2}-\alpha^{2} r_{i}^{n-4}\right) \Omega_{n-4}}{(n-3) \Xi}\right|
$$

and the surface gravity is

$$
\kappa_{i}=\left|\frac{1}{2 \Xi\left(r_{i}^{2}-\alpha^{2}\right)} \frac{d \Delta_{r}}{d r}\right|_{r=r_{i}} .
$$

The action of the brane in (1) can be written in the same form as (21). The total action takes the same form (22).

The relative creation probability, at the $W K B$ level, is

$$
P \approx \exp (-I)
$$

In the probability calculation of the black hole creation, the induced metric and the matter content on the equator of the constrained instanton are given. These constraints can be characterized by a few parameters, like mass $m$, charge $Q$ and angular momentum $J$. Therefore, the path integral can be interpreted as the partition function $Z$ for a microcanonical ensemble in gravitational thermodynamics [14]. In this ensemble, the entropy is

$$
S=\ln Z \approx-I .
$$

The second equality is due to the $W K B$ approximation, in which the path integral is evaluated by the instanton contribution. The neglected contribution is from the fluctuation around the background. There is no contribution from the brane tension.

However, in the braneworld there are two parts in the entropy. One part is due to the tension of the brane. This is new. One may wonder why this phenomenon was not considered in the black hole 
case, say the Schwarzschild-de Sitter case. The reason is that the surfaces of quantum transitions, or the equators for braneworld and black hole creations are different. In the latter case, the 3-metric of the equator is the only configuration for the wave function there, while in the former case, the brane tensions (or the deficit angles) must be included in the configuration. The right choice of the representation is also crucial for dimensionality in quantum cosmology [15].

The other contribution to the entropy is due to the gravity, as in the black hole with a 4dimensional de Sitter background. The result on entropy for the nonrotating higher-dimensional black hole with distinct surface gravities is not new [14]. It is noted that for the black hole entropy one has to replace $\alpha$ by $i a$ in the above formulas, where $a$ is the angular momentum parameter for the real Killing time. In the braneworld the observer is living in a brane. Let us take the nonrotating case with $k=1$ as an example, the brane is a de Sitter spacetime, and the observer perceives his world only through non-gravitational forces, then he would conclude that the universe is created from a $n-2$-dimensional instanton, i.e. $S_{n-2}$ sphere. In the traditional cosmology, the entropy is one quarter of area of the $n-4$-dimensional horizon $S_{n-4}$. In the braneworld the entropy associated with one brane is one quarter of the volume of $S_{n-2}$. In the new scenario, both the two branes and associated tensions contribute to the entropy of the universe.

If one takes the braneworld seriously, one has to evaluate the entropy of the universe in the new way. Since at least one kind of interaction penetrates into the bulk, the $n-2$-dimensional brane is not self-contained. This problem is hidden in the traditional Kaluza-Klein models, since the effect of the extra dimensions to the entropy and the action has been automatically taken into account in redefining the Newton gravitational constant.

From (22) and (29) it follows that the entropy of those braneworlds can take arbitrarily large values as the parameter $|\beta|$, or the tensions, increase. This result of calculation is not only interesting for the braneworld scenario, but also for quantum gravity in general.

There is a new perspective on the origin of the cosmological constant, the so-called " $\Lambda-N$ correspondence" [16]. It is conjectured that the cosmological constant $\Lambda$ is a direct consequence of the finite number of states $e^{N}$ in the Hilbert space describing the world. Many people believe that, in any universe with a positive $\Lambda$ the observable entropy $S$ is less than or equal to $N$. For a given $\Lambda, N$ is saturated by the entropy of de Sitter space, and one has

$$
N=\left[\frac{(n-1)(n-2)}{2 \Lambda}\right]^{\frac{n-2}{2}} \frac{\Omega_{n-2}}{4} .
$$


Bousso is able to show that this is true for all spherical symmetric universes with $n \geq 4$ [16]. He suggested that $\Lambda>0$ may be a sufficient condition for the entropy bound $S \leq N$ without the symmetry condition [17].

However, for $n>4$ case, Bousso, DeWolfe and Myers realized that the conjecture does not hold by providing a counterexample, which is a product space with flux of the form $(A) d S_{p} \times S^{q}[18]$.

The model provided in this letter violates the " $N$-bound" conjecture not only for $n>4$, but also for $n=4$. Since our model is not spherically symmetric, this counterexample does not conflict with Bousso's proof for the spherically symmetric 4-dimensional model [16]. Therefore, it is concluded that, even for $n=4$, the specification of a positive $\Lambda$ is not sufficient to characterize the class of spacetime described by quantum gravity theories with finite-dimensional Hilbert space.

\section{Acknowledgement:}

I would like to thank Xin Xin Du and An Zhong Wang for discussions.

\section{References:}

1. N. Arkani-Hamed, S. Dimopoulos and G. Dvali, Phys. Lett. B $\underline{429}, 263$ (1998); I. Antoniadis,

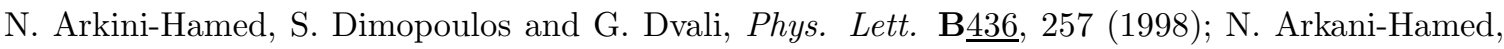
S. Dimopoulos and G. Dvali, Phys. Rev. D $\underline{59}, 086004$ (1999).

2. L. Randall and R. Sundrum, Phys. Rev. Lett. a, 3370 (1999); L. Randall and R. Sundrum, Phys. Rev. Lett. $\underline{83}, 4690$ (1999).

3. P. Horava and E. Witten, Nucl. Phys. B $\underline{460}, 506$ (1996); P. Horava and E. Witten, Nucl. Phys. B $\underline{475}, 94$ (1996).

4. J.B. Hartle and S.W. Hawking, Phys. Rev. D $\underline{28}, 2960$ (1983).

5. Z.C. Wu, Phys. Rev. D $\underline{31}, 3079$ (1985); S.W. Hawking and N. Turok, Phys. Lett. B $\underline{425}, 25$ (1998); N. Turok and S.W. Hawking, Phys. Lett. B $\underline{432}, 271$ (1998).

6.W. Hawking, T. Hertog and H.S. Reall, Phys. Rev. D $\underline{62}$, 043501 (2000); K. Koyama and J. Soda, Phys. Lett. B $\underline{483}, 432$ (2000).

7. J.W. Chen, M.A. Luty and E. Ponton, JHEP 0009, 012 (2000); J.M. Cline, J. Descheneau, M. Giovannini and J. Vinet, JHEP 0306, 048 (2003); P. Bostock, R. Gregory, I. Navarro and J. 
Santiago, hep-th/0311074; R.A. Battye, B. Carter and A. Mennim, hep-th/0312198; H.M. Lee and G. Tasinato, hep-th/0401221; I. Navarro and J. Santiago, hep-th/0402204.

8. E. Witten, Nucl. Phys. B $\underline{195}, 481$ (1982).

9. O. Aharony, M. Fabinger, G.T. Horowitz and E. Silverstein, JHEP 0207, 007 (2002); F. Dowker, J.P. Gauntlett, G.W. Gibbons and G.T. Horowitz, Phys. Rev. Dㅁ2, 6929 (1995); F. Dowker, J.P. Gauntlett, G.W. Gibbons and G.T. Horowitz, Phys. Rev. D포, 7115 (1996).

10. D. Birmingham and M. Rinaldi, Phys. Lett. B $\underline{544}, 316$ (2002); B. Balasubramanian and S.F. Ross, Phys. Rev. D $\underline{66}, 086002$ (2002).

11. See for example, A. Vilenkin and E.P.S. Shellard, Cosmic Strings and Other Topological Defects, Cambridge University Press (1994).

12. R. Gregory, Phys. Rev. Lett. $\underline{84}, 2564$ (2000); I. Olasagasti and A. Vilenkin, Phys. Rev. D $\underline{62}$, 044014 (2000); M. Giovannini, H. Meyer and M. Shaposhnikov, Nucl. Phys. B B19, 615 (2001); S. Kanno and J. Soda, hep-th/0404207.

13. S.W. Hawking, C.J. Hunter and M.M. Taylor-Robinson, Phys. Rev. D $\underline{59}, 064005$ (1999); D. Klemm, V. Moretti and L. Vanzo, Phys. Rev. D $\underline{57}, 6127$ (1998), Erratum-ibid. D $\underline{60}, 109902$ (1999); D. Klemm, JHEP 9811, 019 (1998); M.H. Dehghani, Phys. Rev. D모, 124002 (2002).

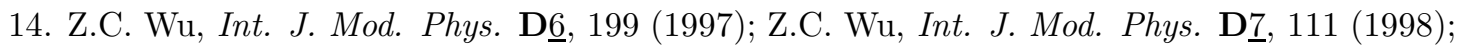
R. Bousso and S.W. Hawking, Phys. Rev. D $\underline{59}, 103501$ (1999), Erratum-ibid. D무, 109903 (1999);

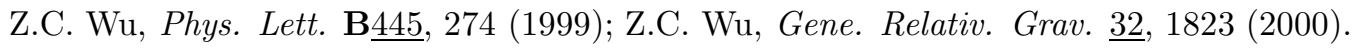

15. Z.C. Wu, Gene. Relativ. Grav. 또, 1121 (2002); Z.C. Wu, Phys. Lett. B $\underline{585} 6$ (2004).

16. W. Fischler, unpublished; W. Fischler, Taking de Sitter seriously. Talk given at Role of Scaling Laws in Physics and Biology (Celebrating the 60th Birthday of Geoffrey West), Santa Fe, Dec. 2000; T. Banks, hep-th/0007146; R. Bousso, JHEP 0011, 038 (2000).

17. R. Bousso, Rev. Mod. Phys. $\underline{74}, 825$ (2002).

18. R. Bousso, O. DeWolfe and R.C. Myers, Found. Phys. $\underline{33}, 297$ (2003). 